In Resolutionen der Europäischen Kommission wird betont, dass ,Lebenslanges Lernen' die Zeitspanne vom Vorschul- bis zum Rentenalter abdecken sollte, wobei das ganze Spektrum von formellem, nicht-formellem und informellem Lernen beachtet werden muss. Fernstudien sind eine Art ,Lebenslangen Lernens'. Der Aufsatz geht der Frage nach, wie Fernstudenten optimal betreut werden könne, was für Materialien z. B. für Fernkurse erforderlich sind und auf welchem Weg diese Materialien zu den Studierenden gelangen. Welche Fragestellungen und Aufgaben kommen auf die Bibliotheken, auf die Bibliothekare und auf die Bibliothekswissenschaft in diesem Kontext zu?

\title{
Bibliotheksservice für Fernstudenten
}

"Knowledge is of two kinds. We know a subject ourselves, or we know where we can find information upon it." Samuel Johnson, 1709-1784

\section{Einführung}

Fast jeden Tag können wir von unseren Bibliotheksbesuchern hören: „Wie schade, dass wir dies nicht schon vorher lernen durften!“ Wir hören es sowohl von Studenten, die auf dem Campus studieren als auch von Fernstudenten. Sie sprechen mit dieser Aussage den Prozess des Informationssuchens und der Literaturrecherche an, denn sie haben feststellen können, dass ,Informationskompetenz' nicht das gleiche ist, wie das Internet absuchen zu können.

In diesem Beitrag möchte ich einige Hinweise geben, was man bedenken muss, wenn man Studenten helfen will - entweder direkt von einer Universitätsbibliothek aus, direkt oder indirekt durch eine öffentliche Bibliothek oder auch mit Hilfe eines ,Lernzentrums ‘. ${ }^{\prime}$

Durch die Zusammenarbeit in einem Team von Studierenden, Lehrern, Bibliothekaren, Technikern etc. erfahren und erproben alle Beteiligten Strukturen und Techniken der Wissensvermittlung. Diese Kenntnis ist wichtig: Erstens, um sich selber verstehen zu können, und zweitens, um andere zu verstehen und um ihnen dann zu helfen darüber zu reflektieren, wie Bildung, Ausbildung und Lernen in unserer Gesellschaft entsteht. Für Bibliothekare ist

\footnotetext{
${ }^{1}$ Im Englischen hat sich der Begriff "Learning Resource Center" eingebürgert.
} 
deshalb - neben dem Studium der Bibliotheks- und Informationswissenschaft - auch eine pädagogische Ausbildung erforderlich.

Die Bibliothek ist ein wichtiger gesellschaftlicher Ort. Sie ist ein Ort der Informationsvermittlung und der Wissensbildung, offen für jedermann. Allerdings, wie ein schottisches Sprichwort sagt: „Man kann ein Pferd zu einer Wasserquelle führen, aber man kann es nicht zwingen zu trinken.“2

Die Person, die den Bibliotheksbesuchern helfen kann, Informationen zu finden, um ihr Wissen zu erweitern, muss ein gut ausgebildeter und kompetenter Bibliothekar sein. Für die Kompetenz der Bibliothekare, die in der Informations- und Wissensgesellschaft der Zukunft arbeiten sollen, haben wir an der Universität in Växjö, Schweden, ein drei Jahre währendes akademisches Studium eingeführt. Ein Schwerpunkt der Studien ist Pädagogik.

\section{Hintergrund}

Die schnelle Entwicklung der Informations- und Kommunikationstechnologie (IKT) und die steigende Zahl der Studenten an unseren Universitäten und Fachhochschulen während der letzten Jahre hat die Rolle der Bibliotheken und die der Bibliothekare dramatisch verändert. Nicht dass Bibliotheken plötzlich verwaist wären (nach dem Motto, ,es gibt ja alles im Internet“), im Gegenteil, mehr und mehr Menschen versammeln sich in Bibliotheken (zumindest in Schweden) um Information zu suchen und in Gruppen zu arbeiten.

Sie verlangen längere Öffnungszeiten, mehr Bücher und anderes Material in den Regalen, mehr Computer, mehr elektronische Medien usw. In den 1990er Jahren haben sich die schwedischen Universitätsbibliotheken fundamental verändert. Während sie zuvor in erster Linie als Wissenschaftsbibliotheken den etablierten Forschern dienten, wandelten sie sich ziemlich plötzlich in Studienbibliotheken für Studenten, wobei sie immer noch auch den Forschern Service anbieten mussten. Bereits dies zog verschiedene Konsequenzen nach $\operatorname{sich}^{3}$, und außerdem kamen noch die berechtigten Wünsche der Fernstudenten hinzu. Es wurden Fragen aufgeworfen wie: „Hat jeder die gleichen Rechte und Möglichkeiten, an elektronisches Material heranzukommen, egal an welcher Universität oder an welchem Ausbildungsinstitut er studiert, wo er lebt und zu welcher Art Bibliothek er geht?“ - „Welche Bedeutung haben die IKTKenntnisse dieses Benutzers, welche Bedeutung hat sein sozialer oder ökonomischer Status, damit er überhaupt an elektronischen Fernkursen teilnehmen kann?"

\footnotetext{
2 "You can lead a horse to water well, but you can't force it to drink!"

3 BIBSAM (1995-1996); Gellerstam (2002).
} 
Fragen wie diese haben Jakob Harnesk in einer Studie beschäftigt, die er im Auftrag unserer „Authority of Distance Education in Sweden“ (wir nennen sie kurz „Netzuniversitätsbehörde“4 ) durchgeführt hat. ${ }^{5}$ Auch für andere Bibliothekare erwiesen sich diese Fragen als wichtig. ${ }^{6}$

Die schwedische sog. „Netzuniversität“ wurde im November 2001 gegründet. Diese ,Universität ${ }^{\star}$ ist ein Netzwerk aller schwedischen Universitäten und Fachhochschulen, die elektronische Kurse über das Internet anbieten. Es gibt also keinen physischen Platz, wo ein Student hingehen kann oder muss, um z. B. Vorlesungen folgen zu können.

Man geht davon aus, dass die Studenten entweder von zu Hause aus über den eigenen Rechner studieren oder zu verschiedenen Lernzentren gehen, auch zur nächsten öffentlichen Bibliothek, oder dass sie Kurse belegen, die von anderen Universitäten als derjenigen, wo sie immatrikuliert waren, angeboten werden. Sie sind dann Studenten der Netzuniversität. Ein Gremium für allgemeinen Service für diese Studenten, einschließlich Bibliotheksservice, wurde bald gegründet. Der Direktor der Netzuniversität, Dr. Mats Ericson, sagte in einem Interview ${ }^{7}$, dass er sich wünschte, dass Lehrer, Bibliothekare und Techniker in Teams arbeiten sollten, um den Studenten die optimale Hilfe geben zu können, und um überhaupt die höhere Fernausbildung reformieren zu können.

\section{Warum Bibliotheken im modernen Unterricht}

Die höheren Ausbildungen werden sowohl lokal von einer Universität als auch über das Internet angeboten. Dabei kann es sich sowohl um Fernstudien als auch um Campus-Studien handeln. Die Studienangebote über das Internet entwickeln sich mit zunehmender Geschwindigkeit. Deshalb werden die höheren Studien immer mehr ein Teil des lebenslangen Lernens werden, und mehr und mehr Menschen werden auch an höheren Internet-Kursen teilnehmen, wenn sie ihre grundlegende Ausbildung bereits absolviert haben und inzwischen im Berufsleben stehen.

Studierende aller Art werden ihre Kurse zusammenstellen, verteilt auf mehrere Universitäten oder Institute, sowohl aus ihren eigenen Ländern als auch aus dem Ausland. Für Fernstudenten ist es oft problematisch, korrekte Information $\mathrm{zu}$ erhalten, die für ihre Studien relevant sind und die sie brauchen, um erfolgreich studieren zu können. Oft genug leben sie weit weg

\footnotetext{
${ }_{5}^{4}$ Siehe: http://www.netuniversity.se.

5 Harnesk (2002).

${ }_{7}^{6}$ Z. B. das DERAL-Projekt, unterstützt von der EU im 4. Rahmenprogramm.

${ }^{7}$ Ericson (2002).
} 
vom eigenen Campus und können keinen Zugang zu Information bekommen, die für ihre Kommilitonen an der eigenen Universität selbstverständlich und ohne Mühe erreichbar sind.

Wissenschaftliche und andere Bibliotheken müssen sich auf diese Veränderung einstellen. Für sie bedeutet das z. B.:

- erweiterte Öffnungszeiten - an manchen Universitäten schon der 24/7 Service (geöffnet 24 Stunden pro Tag, 7 Tage die Woche),

- mehr und mehr Selbstbedienung für Bibliotheksbesucher,

- mehr Hilfe zur Selbsthilfe durch Unterricht in Information Literacy. Dieser Unterricht wird von Bibliothekaren erteilt und auf allen Ebenen gegeben, von den neu immatrikulierten Studenten bis hin zu Forschern und Professoren. Dies bedeutet, dass Bibliothekare gut ausgebildet sein müssen sowohl in Bibliotheks- und Informationswissenschaft, als auch in Pädagogik,

- mehr entwickelter Service in der Informations-Technik (IT), da heutzutage nicht nur die Kataloge online sind, sondern auch Datenbanken, elektronische Zeitschriften, Arbeitspapiere, wissenschaftliche Artikel, Doktorarbeiten - entweder als Abstracts oder in Volltext -, e-Books, Link-Sammlungen, Portale etc.,

- $\quad$ eLearning ist ebenfalls ein Konzept für viele neue Studenten. ${ }^{8}$ Häufig genug geschieht dieses Lernen in einer Bibliothek, und deshalb müssen die Bibliotheken so ausgerüstet werden, dass sie den aktuellen Entwicklungen gerecht werden und folgen können.

Diese Veränderungen wurden notwendig, weil sich, zumindest in Europa in den letzten Jahrzehnten, sowohl die Anzahl als auch die Zusammensetzung der Studenten stark verändert hat. Bedeutend mehr Studenten als früher nehmen heute an allen möglichen Kursen teil. Das Ziel unserer schwedischen Regierung ist, dass $50 \%$ eines jeden Jahrganges an einer Universität oder Fachhochschule immatrikuliert werden soll. In manchen Regionen ist dieses im Jahr 2004 bereits erfüllt. $^{9}$

\footnotetext{
${ }^{8}$ Siehe z. B. http://www.content-village.org oder http://www.elearningeuropa.info beide von der Europäischen Kommission unterstützt.

${ }^{9}$ Siehe: http://www.hsv.se
} 
Hinzu kommt, dass die Unterrichtspädagogik sich sehr verändert hat. Man möchte, dass Studenten in ihrem Lernen sehr viel aktiver sein sollen als früher. Diese sich verändernden Muster können kurz wie folgt zusammengefasst werden:

- Weniger junge Studenten. Wenn immer weniger Kinder geboren werden, wird die Anzahl der jungen Studenten, also zwischen etwa 18 und 25 Jahren, kleiner werden. Außerdem möchten viele junge Menschen nach der Schule erst eine ,Pause' machen und sich ,in der Welt umschauen', sei es zum Meditieren in den Himalajas oder zum Tauchen am Great Barrier Reef, ehe sie sich dem ,Ernst des Lebens“ widmen und anfangen, für die Zukunft zu studieren.

- Mehr ältere und schon berufstätige Studenten. Je mehr verschiedene Arbeitgeber von ihren Angestellten verlangen, dass sie über den letzten Stand der Entwicklung informiert sein sollen, umso mehr ältere Studenten werden zusammen mit jüngeren studieren wollen, besonders als Fernstudenten. ${ }^{10}$ Viele von diesen gehen zuerst zu ihrer nächsten Bibliothek, d. h. zu ihrer öffentlichen Bibliothek, um Hilfe zu suchen. Bibbi Lundborg ${ }^{11}$ untersuchte schon im Jahr 2000 in einer Studie, wie viele Besucher in einer öffentlichen Bibliothek eigentlich Studenten waren, und konnte feststellen, dass ganze $50 \%$ sich als Studenten mit besonderen Anforderungen an den Service bezeichneten. Auch an unserer Universität haben wir gesehen, dass Fernstudenten öfter älter und öfter weiblich sind, und wir vermuten (ohne es jedoch bis jetzt verifiziert zu haben), dass Fernstudien attraktiv sind für Menschen, die bislang keine Möglichkeit hatten zu studieren, da sie zu den sog. ,unteren Sozialgruppen' gehören.

- Mehr Variation in vorherigem Wissen. Wenn ältere Menschen zusammen mit jüngeren an Kursen teilnehmen, ist von unterschiedlichen Vorkenntnissen auszugehen - sowohl in der Theorie als in den praktischen Erfahrungen. Allerdings, wenn nun mehr und mehr Menschen aus den unteren Sozialgruppen an höheren Studien teilnehmen, ohne Unterstützung z.B. von einschlägig erfahrenen Familienmitgliedern zu bekommen, dann sind auch ihre Schwierigkeiten den Studien zufolge ganz anders als bei Studierenden, die in ihrem Umfeld schnell Verständnis und Hilfe bekommen können.

\footnotetext{
${ }^{10}$ In Växjö waren 2003 über $20 \%$ aller Studenten an der Universität über 30 Jahre alt.

${ }^{11}$ Lundborg in Kommunaktuellt (2000).
} 
Welche Probleme dies für die Lehrer und für das Bibliothekspersonal mit sich bringt, kann man nur ahnen.

- Kombination von Arbeit und Studien. Wenn die Tendenz zum lebenslangen Lernen weiter ansteigt, wird es sicher immer mehr üblich werden, dass Menschen ihre Berufsarbeit mit Studien kombinieren wollen. Dies müsste von der Europäischen Kommission und von den nationalen Regierungen (und das nicht nur in Europa) beachtet werden, sodass Menschen, die studieren wollen, dies auch wirklich tun können.

- Kulturelle Vielfalt. Nicht nur, aber speziell die jüngere Generation, reist heute oft umher und findet dadurch vielleicht ganz andere Orte, wo sie (zumindest eine Zeit lang) wohnen und leben möchte, als am Geburtsort. Viele und große Flüchtlingsströme haben die Bevölkerungskarte in Europa zum Teil neu gezeichnet. An manchen Orten gibt es leider viele Probleme, an anderen hat man die kulturelle Vielfalt als etwas Positives gesehen und versucht, sich einzurichten und neue Unterrichtsmethoden zu entwickeln. Probleme, wer was wann wo und in welcher Sprache studieren darf und kann, konnten gelöst werden. Auch hier waren oft genug die Bibliotheken an der Lösung dieser Probleme beteiligt.

- Größere Beweglichkeit. Studenten haben eine Vielfalt von Möglichkeiten, gleichzeitig an verschieden Universitäten und anderen Instituten im In- und Ausland zu studieren. Und sie tun es! Oder sie ziehen in der realen Wirklichkeit von einer Universität zur nächsten und studieren mal hier und mal da etwas.

- Bessere IKT-Kenntnisse. Wie ist es nun mit der Informations- und Kommunikationstechnologie? Immer mehr Menschen lernen immer schneller, wie die Informations- und Kommunikationstechnologie funktioniert. Viele Schulen geben ihren Schülern schon eigene PCs, sowohl Grund- als auch höhere Schulen. Aber die Technik bringt auch Probleme, sowohl pädagogische als auch rein technische. Verschiedene Datenbanken verlangen verschiedene Suchtechniken, die nicht jedermann beherrscht. Viele verstehen auch nicht den Unterschied zwischen ,das Richtige zu finden' und ,überhaupt etwas zu finden'. Die Europäische Kommission hat betont, wie wichtig es ist, dass junge Leute Informationskompetenz erwerben, und hat auch viel Geld investiert, um in Schulen Internet-Anschlüsse $\mathrm{zu}$ installieren. Tatsächlich haben immer mehr Menschen eingesehen, dass der Prozess 
der Informationssuche und die neuen Wege, miteinander zu kommunizieren, schwierig zu erlernen sind und es nicht mit der rein technischen Verkabelung getan ist.

\section{Die Europäische Dimension}

Die Europäische Kommission hat mehrere Aussagen über Ausbildung und lebenslanges Lernen gemacht (z. B. März 2000 in Lissabon, März 2002 in Barcelona) - und Fernstudien sind eine Möglichkeit des lebenslangen Lernens. Die Kommission betont, dass lebenslanges Lernen das ganze Lern-Spektrum vom Vorschul- bis ins Rentenalter abdecken muss. Es soll auch sowohl formelles wie auch informelles und nicht-formelles Lernen abdecken. ${ }^{12}$ Und wieder kommen die Bibliotheken ins Spiel, denn: wie sollen diese Menschen ihre berechtigten Wünsche erfüllt bekommen, wenn sie verschiedenes Material brauchen, um an Fernkursen und lebenslangem Lernen teilzunehmen, wenn nicht von ihren nächstgelegenen Bibliotheken, wo gut ausgebildetes und kompetentes Personal ihnen weiterhilft?

\section{Wie definiert man einen Fernstudenten und weitere Fragen}

Einen Fernstudenten zu definieren ist nicht so leicht und eindeutig, wie man zunächst annehmen könnte. Ist es:

- ein Student, der an einem Studieninstitut eingeschrieben ist, aber zu Hause weit weg von diesem Institut lebt und nur elektronisch über das Internet am Studium teilnimmt?

- ein Student, der an Kursen teilnimmt, die das Institut als Fernkurse anbietet, an denen man aber persönlich mehr oder weniger oft zu gewissen Präsenzzeiten teilnehmen muss? Falls ja, wie oft muss der Student persönlich kommen, damit er als Fernstudent registriert wird? Einmal pro Semester, zweimal im Monat oder noch öfter? Vielleicht überhaupt nicht?

- ein Student, der zusätzlich an einem anderen Institut studiert neben dem, an dem er immatrikuliert ist?

\footnotetext{
12 Siehe: http://europa.eu.int/comm/education/policies/111/111_en.html; The Strategies in Life Long Learning Projekt (SILL_NET): http://www.sillnet.com/welcome.html
} 
- ein Student, der ,nur noch' seine Prüfung vorbereitet und das Institut eigentlich schon verlassen hat?

- ein Student, der zu gleicher Zeit verschiedenen Kursen an verschiedenen Instituten / Universitäten folgt?

Andere Fragen, die sich im Lauf der Zeit ergaben, lauten z. B.:

- Sollte der Service der nächsten erreichbaren (wissenschaftlichen) Bibliothek kostenfrei sein, oder sollten Fernstudenten dafür bezahlen? Falls ,ja“, warum, und wie viel?

- Soll es einen Unterschied zwischen den verschiedenen Studentenkategorien geben in der Hinsicht, wie ihnen geholfen wird und wie viel sie evtl. bezahlen müssen?

- Und wie ist es mit den öffentlichen Bibliotheken? Wir wissen, dass viele Studenten tatsächlich zu ihrer nächsten öffentlichen Bibliothek gehen, um dort Hilfe und Service zu bekommen. ${ }^{13}$ Nur - die meisten öffentlichen Bibliotheken haben keinen Zugang zu wissenschaftlichen Datenbanken und elektronischen Zeitschriften. Wie kann also diesen Studenten geholfen werden, und wie können oder müssen diese Bibliotheken finanziell ausgestattet werden?

- Welche Rolle spielen hier die sog. ,Lernzentren“, die überall in Schweden entstehen?

- Ist die Ausbildung des Bibliothekspersonals gut genug, damit die Bibliothekare diesen verschiedenen Arten von Studierenden helfen können?

- Ist die Kompetenz in Information Literacy hoch genug sowohl bei Lehrern als auch bei den Bibliothekaren und Studenten, damit sie die verschiedenen Suchprozesse lernen und verstehen können? Falls „Nein“, wie kann man die Kompetenz verbessern?

Es ist offensichtlich wichtig, dass nicht nur Bibliothekare, sondern auch Lehrer und vor allem leitende administrative Personen an unseren Universitäten und

${ }^{13}$ Kühne (2001). 
den übrigen Ausbildungsinstituten zusammenarbeiten und eine gemeinsame Strategie entwickeln, wie man diese Probleme lösen könnte.

\section{Bibliotheksservice für Fernstudenten}

Jakob Harnesk, Bibliothekar und ehemaliger Kollege von BIBSAM ${ }^{14}$, heute bei BTJ ${ }^{15}$ tätig, hat im Auftrag von BIBSAM im Jahr 2002 in einer groß angelegten Studie untersucht, wie tatsächlich der Bibliotheksservice für schwedische Fernstudenten funktioniert. Auch wir an der Växjöer Universität nahmen daran teil. Daraus ergab sich ein neues Projekt zusammen mit der Stockholmer Universität, wo näher untersucht wird, wie man die sog. ,Studienzentren' mit den vorhandenen Bibliotheken verbinden kann oder soll. ${ }^{16}$ Eine andere Studie zu diesem Thema wurde von Helen Hed von der Universitätsbibliothek in Umeå durchgeführt. ${ }^{17}$

Im Jahr 2003 bekam die Universitätsbibliothek in Jönköping Mittel von der Netzuniversität, um das Projekt mit dem „Diensthabenden Bibliothekar“ zu starten (engl.: Librarian on Duty ${ }^{18}$ ). Dieses Projekt bedeutete, dass Studierende aller Art, jedoch hauptsächlich Fernstudenten, in den Genuss des 24/7-Service kamen. D. h. sie konnten rund um die Uhr ihre Fragen stellen und Hilfe bekommen. Nicht, dass sie direkte Antworten auf ihre Fragen bekamen, nein, sie wurden (elektronisch) z. B. zum Katalog geführt, zu den Datenbanken, zu den elektronischen Zeitschriften, und man half ihnen (immer noch elektronisch), das zu suchen und zu finden, was sie brauchten.

Das Programm erlaubt auch sog. Co-browsing: Der Leiter des Projekts, UlfGöran Nilsson, fand heraus, dass die meisten Studenten einen ,richtigen“ Bibliothekar bevorzugten, (auch wenn dieser eigentlich nur elektronisch erreichbar war) und weniger Interesse zeigten, einen vollständigen RechercheKurs über das Internet zu erhalten. Beim Co-browsing kann der Fragende den Rechercheprozess, den der Bibliothekar an seinem Monitor vollführt, am eigenen Monitor - an einem beliebigen Ort oder auch dem Bibliothekar direkt gegenüber sitzend - mit-, lesen“.

Die öffentlichen Bibliotheken haben ein ähnliches Projekt durchgeführt ${ }^{19}$, und jetzt ist man dabei, eine technische Brücke zwischen diesen beiden Systemen zu bauen, sodass der Suchende gar nicht merken wird, in welchem

\footnotetext{
${ }^{14}$ Eine Abteilung an unserer Nationalbibliothek: http://www.kb.se/BIBSAM.

${ }_{15}^{15}$ Bibliotekstjänst, ein schwedisches Bibliotheksunternehmen: http://www.btj.com.

${ }_{17}^{16}$ Start 2003, läuft bis 2005. Siehe: http://www.netuniversity.se.

${ }_{18}^{17}$ Hed (2002).

${ }_{19}^{18}$ Siehe: http://www.netuniversity.se/jourbibliotekarie.

19 "Fråga biblioteket", siehe: http://www.vuxbib.nu.
} 
System er sich befindet und welche Art Bibliothekar ihm die Antwort vermittelt.

\section{Probleme mit dem Bibliotheksservice für Fernstudenten}

Die folgenden Abschnitte sind hauptsächlich den Studien von Jakob Harnesk ${ }^{20}$ und Helen $\mathrm{Hed}^{21}$ entnommen, aber es fließen auch eigene Erfahrungen und Untersuchungen mit ein. ${ }^{22}$

\subsection{Literaturbeschaffung und Ausleihe}

Auch wenn immer mehr Literatur in elektronischer Form angeboten wird, ist immer noch das Buch eine wichtige Quelle für Studenten aller Art, um ein erfolgreiches Studium absolvieren zu können. Dies gilt insbesondere für diejenigen, die nicht gewöhnt sind, mit Computern und dem Internet umzugehen, oder die z. B. von zu Hause aus keinen direkten Zugang zum Internet haben.

Die meisten schwedischen wissenschaftlichen Bibliotheken senden gedrucktes Material kostenlos zu den Bestellern, aber diese müssen es auf eigene Kosten zurückschicken (und Bücher sind schwer, daher sind Portokosten sehr hoch!).

Die meisten öffentlichen Bibliotheken verlangen andererseits, dass ein Besteller das Bestellte persönlich abholt. Langsam setzt sich durch, dass manche Regionen es zulassen, dass bestelltes Material auch von den öffentlichen Bibliotheken direkt an den Besteller geschickt wird, und dass dieser es dann bei der nächsten Bibliothek kostenlos abgeben kann, und es dann von dieser aus an die Ursprungsbibliothek zurückgeschickt wird.

Andere Versuche laufen darauf hinaus, dass einige öffentliche Bibliotheken Zugang zu den am meisten frequentierten Datenbasen der wissenschaftlichen Bibliotheken bekommen sollen (KULDA-Projekt ${ }^{23}$ ). Hier sind jedoch die Lizenzproblematik und die Kosten, die damit verbunden sind, noch nicht geklärt.

\footnotetext{
${ }^{20}$ Harnesk (2002).

${ }^{21} \mathrm{Hed}$ (2002).

${ }^{22}$ Kühne (2001) und späteres, noch nicht veröffentlichtes Material.

${ }^{23}$ Siehe: http://www.kulda.se.
} 


\subsection{Zugang zu Datenbanken und elektronischen Zeitschriften}

Heutzutage bieten die meisten wissenschaftlichen Bibliotheken Zugang zu wissenschaftlichen Zeitschriften und Datenbanken, auch wenn der Student nicht persönlich in die Bibliothek kommt. Er erhält dann von jedem beliebigen Ort aus den Zugang über einen Proxy Server und einen persönlichen Bar Code (oder ein ID-log-in-password) und gelangt so über das Internet an das elektronische Material seiner Universitätsbibliothek. Einzige Bedingung dabei ist, dass dieser Bar Code wirklich persönlich sein muss, und der Student muss an dieser Universität immatrikuliert sein, um den Code zu bekommen.

In Jönköping hat man es so gelöst, dass der Student automatisch, wenn er sich immatrikuliert, eine Bibliothekskarte bekommt, die ihm dann ermöglicht, über den Proxy Server das elektronische Material zu erreichen. Das ist eine elegante und einfache, letztlich übertragbare Lösung, die natürlich auch anderswo praktiziert werden könnte. ${ }^{24}$

\subsection{Ausbildung zur Vermittlung von Information Literacy}

Im Jahr 2001 beschloss die schwedische Regierung ${ }^{25}$, dass InformationsKompetenz eines der wichtigsten Ziele für alle Ausbildungsgänge an schwedischen Universitäten und Fachhochschulen sein sollte. Ausdrücklich wird im Hochschulgesetz gesagt (Übers. der Verf.): „Jeder Student soll imstande sein, Wissen auf einem wissenschaftlichen Niveau selbständig suchen und auf seine Gültigkeit hin überprüfen zu können. “26

Diesem Gesetz folgend bieten alle Bibliotheken an schwedischen Universitäten und Fachhochschulen heute ihren Studenten Kurse in „Information Retrieval und Information Literacy“ an, entweder individuell oder gruppenweise, sowohl für Studenten auf dem Campus als auch für Fernstudenten, wenn diese sich z. B. besuchsweise an der Universität aufhalten.

Manche Bibliotheken, wie unsere, haben einen speziellen ,Kontaktbibliothekar' für Fernstudenten, und durch diesen können die Studenten sehr detaillierte und individuell zugeschnittene Hilfe bekommen.

Andererseits: Die Anzahl der Fernstudenten steigt immer mehr - schon jetzt sind mehr als $20 \%$ der etwa 15000 immatrikulierten Studenten an der Universität in Växjö sog. Fernstudenten! Mit anderen Worten: über 2000 Personen! Wie soll ein einziger Kontaktbibliothekar allen diesen helfen können?

\footnotetext{
${ }^{24}$ Siehe: http://www.bibl.hj.se.

25 Öppna högskolan (2002).

${ }^{26}$ SFS 2003:1121, Kap 1, §9.
} 
In diesem Jahr (2004) haben wir deshalb einen internet-basierten „Sök (Such-) Guide“" auf unsere Homepage gesetzt. ${ }^{27}$ Es handelt sich dabei um eine Art elektronischen Kurs in Information Literacy. Dieser Kurs hilft Studenten, sowohl denen auf dem Campus als auch den Fernstudenten, ihre verschiedenen Fragen zu beantworten. Selbst wenn es keine wirkliche Person ist, wie bei dem diensthabenden Bibliothekar, der die Fragen direkt beantwortet, haben wir doch gesehen, dass er eine große Hilfe für die Studenten ist. Wir hoffen, den Kurs für unsere ausländischen Studierenden bald auch in englischer Sprache anbieten zu können.

\subsection{Entwicklung pädagogischer Kompetenz}

Wenn Bibliothekare immer öfter ihre neue Rolle betonen, nicht nur Information und Material für ihre Bibliotheksbesucher zu finden, sondern die Bibliotheksbenutzer auch dahingehend zu schulen, ihre Recherchen selbst durchzuführen, dann ist es auch wichtig, dass Bibliothekare in einer pädagogischen Ausbildung entsprechende Kompetenz erwerben. ${ }^{28}$

So gibt es z. B. einen großen Unterschied im Unterrichten von Kindern im Vergleich zu Erwachsenen. Ebenso wichtig ist es, dass wissenschaftliche und öffentliche Bibliotheken zusammenarbeiten, dass die verschiedenen Personalkategorien an beiden Bibliothekstypen von einander wissen - wie sie arbeiten, welche Unterschiede es in den Zielgruppen gibt, wie die ökonomischen Strukturen aussehen, welche Prioritäten gesetzt sind etc.

Allerdings, schwedische Bibliothekare haben selten eine Ausbildung in Pädagogik. Aus diesem Grund hat man in Växjö im Herbstsemester 2003 eine ganz neue Bibliothekarausbildung gestartet, die über sechs Semester läuft und mit einem Mag. Phil. in Bibliotheks- und Informationswissenschaft endet. Schwerpunkte in den Studien sind natürlich Bibliotheks- und Informationswissenschaft, aber auch Pädagogik und Wissenstheorien: wie Wissen gebildet wird, wie es reflektiert wird und dergl. mehr. Schließlich müssen die Studierenden lernen, was unter dem Begriff ,Wissen' subsummiert wird, wenn z. B. in der EU von einer, Wissensgesellschaft " gesprochen wird.

Ergänzend ist es äußerst wichtig, dass auch Lehrer - aller Schularten und auf allen Ebenen - ihre Kenntnisse über Bibliotheken und deren Angebote und Leistungen verbessern.

(Da ich selbst in verschiedenen Stadien meines Lebens auf Fernstudien angewiesen war, möchte ich gerne noch hinzufügen, dass es gar nicht unwichtig ist, über eigene Erfahrungen zu diesem Phänomen zu verfügen. Man

\footnotetext{
${ }^{27}$ Siehe: http://www.bib.vxu.se/sokguide.

${ }^{28}$ Kühne (1993).
} 
versteht die Schwierigkeiten besser, wenn man selbst Student in einer abgeschiedenen ,Wildnis“ gewesen ist: Allein, fern von interessanten Diskussionen mit Kommilitonen oder Forschern, und ohne schnelle Hilfe, sodass man am liebsten aufgeben möchte.)

\subsection{Kooperation zwischen Lehrern und Bibliothekaren}

In der Studie von Jakob Harnesk ${ }^{29}$ haben fast alle an der Studie teilnehmenden Bibliothekare betont, wie wichtig und zugleich schwierig es ist, zu einer besseren Zusammenarbeit mit den Hochschullehrern zu kommen. Ich habe das Problem bereits 1993 in meiner Dissertation thematisiert, doch es hat sich offensichtlich seitdem nicht viel geändert.

Mit dem oben genannten Projekt zusammen mit der Universität von Stockholm im Auftrag der Netzuniversitätsbehörde hoffen wir allerdings bald zu einer Lösung zu kommen, denn wir glauben, dass ein Fernkurs nur dann wirklich effizient sein kann, wenn die Bibliothek von Anfang an in die Planung des Kurses einbezogen wird, sodass die Studenten wissen, wie und wo sie ihr Kursmaterial finden und bekommen können.

\subsection{Kooperation zwischen wissenschaftlichen und öffentlichen Bibliotheken und Lernzentren}

Für viele Fernstudenten (aber auch für andere) haben sich die öffentlichen Bibliotheken und die sog. Lernzentren (engl. Learning Centers oder Learning Resource Centers) zu Treffpunkten entwickelt, wo sie sich wohlfühlen, wo sie mit Gleichgesinnten Gedanken austauschen und wo sie Hilfe bekommen können von geschultem (und meistens sehr freundlichem) Personal.

Sogar technische Hilfe wird ihnen oft zuteil, wenn sie z. B. zu Hause über keinen eigenen Computer verfügen oder mit der Bedienung Probleme haben. Besonders Studierende aus nicht-akademischen Kreisen sind auf diese Hilfe angewiesen.

Die schwedischen öffentlichen Bibliotheken haben eine lange Tradition, allen Menschen zu helfen. Akademische Studenten haben allerdings erst in neuester Zeit ihren Weg in so großen Scharen, wie jetzt, zu diesen Bibliotheken gefunden.

Manchmal werden auch Lernzentren nach angloamerikanischem Modell gebaut, leider oft separiert von öffentlichen Bibliotheken und ohne diese davon zu informieren, aber mit dem gleichen Ziel, nämlich Studierende aller Art bei ihren Recherchen und mit Kursmaterial zu helfen, und ihnen Platz zu bieten

${ }^{29}$ Harnesk (2002). 
für Studien, Diskussionen, mit Computern ausgerüstet usw. Personalmangel gibt es allerdings hier wie dort. Es wäre sicher ökonomisch sehr viel effizienter, wenn man zusammenarbeiten würde, und dort, wo es möglich ist, die Lernzentren mit den öffentlichen Bibliotheken zusammenlegen würde. Die schwedische Organisation NITUS ${ }^{30}$ untersucht dies zur Zeit und nennt gute Beispiele, wie man das machen kann oder auch wo diese Form der Zusammenarbeit nicht angebracht ist.

\subsection{Internationale Guide Lines}

International werden diese Probleme ebenfalls seit längerem diskutiert. Die besten Guide Lines für Information Literacy Competence sind, soweit mir bekannt ist, diejenigen der ACRL (Association of College and Research Libraries), 2004 revidiert. $^{31}$

\section{Fazit}

Ich möchte mit den folgenden Überlegungen schließen:

- Elektronische und Multimedia-Materialien werden sich weiterhin sehr rasch verbreiten und vermehren, sowohl durch e-Publishing als auch durch Einkauf aus anderen Quellen.

- Bibliotheken werden sehr viel mehr in die akademischen Studiengänge integriert werden müssen, zumindest in die grundlegenden Studiengänge. Forscher haben dann später, nach meinen Erfahrungen, oft ganz andere Netzwerke und sind nicht in dem gleichen Ausmaß wie Studenten auf Bibliotheksunterstützung angewiesen. Allerdings möchten sie die gleichen elektronischen Materialien in ihren eigenen Forschungsräumen haben, wie sie sie in der Bibliothek finden können. Dass die Bibliotheken für die Kosten dieser Medien aufkommen, ist den meisten Forschern gar nicht bewusst.

- Die Rolle der Lehrer und der Bibliothekare überschneidet sich teilweise. Im Idealfall arbeiten beide Seite an Seite mit vollem Respekt für die Eigenart und die Eigenkompetenz des anderen. Die Bibliothekar-Ausbildung muss dieses beachten und Pädagogik ebenso

\footnotetext{
${ }^{30}$ Siehe: http://www.nitus.org.

31 Mehr Informationen siehe: http://www.ala.org/ala/acrl/acrlstandards/ informationliteracycompetency.htm.
} 
wie die Didaktik der Wissensvermittlung in ihre Ausbildung integrieren.

- Bibliotheken werden in Zukunft immer mehr spezifische Aufgaben übernehmen, wie z. B. technischen oder editorischen Support, der Hilfe beim Schreiben, beim Layout, beim Druck oder auch beim Publizieren bietet.

- Die Bedeutung des physischen Raumes ,Bibliothek“ wird immer wichtiger. Schon heute sehen wir an unserer Universität, wie sehr die Studenten klagen, dass nicht genügend (größere und kleinere) Gruppenarbeitszimmer zur Verfügung stehen. Viele unserer Kurse gehen nach der PBL-Methode (Problem Based Learning) vor, was wiederum bedeutet, dass die Bibliothek nicht nur ein Platz zum Lernen ist, sondern auch ein Platz für gemeinsame Studien, ein Treffpunkt, ein Platz für Diskussionen und vielleicht sogar ein Platz für neue, innovative und kreative Ideen.

Für die Zukunft werden Bibliotheken Arbeitsweisen entwickeln müssen, mit denen sie den Herausforderungen der Zukunft begegnen können: Entwicklungen wie das zunehmende Life-long Learning oder die Strukturveränderungen bei den Studierenden der Higher Education bringen Anforderungen hinsichtlich der Medien und der Räume, aber auch hinsichtlich des Personals und seiner Kompetenzen mit sich. Die bibliothekarischen Ausbildungsinstitute werden sich dem zu stellen haben. Wissenschaftlich fundierte Forschung sollte diesen Prozess begleiten, indem z. B. Best-Practice Studien erstellt und ausgewertet werden und die weitere Entwicklung z. B. mit Hilfe bibliothekswissenschaftlicher Messmethoden rechtzeitig vorausschauend beobachtet und analysiert wird.

\section{Literatur und Internetquellen}

ACRL: Guidelines for distance learning library service. Chicago, Association of College and Research Libraries, 2004, http://www.ala.org/ala/acrl/ acrlstandards/informationliteracycompetency.htm.

BIBSAM: Studenternas bibliotek. Stockholm 5 (1995/96)

BIBSAM at the Royal Library (KB), http://www.kb.se/BIBSAM

Ericson, Mats: Biblioteksbladet 87 (2002) 5, S. 18-19

European Commission, http://europa.eu.int/comm/index_en.htm 
Gellerstam, Göran: Den första uppgiften: högskolebiblioteket som utbildningsinstitution och lärande miljö, http://www.kb.se/BIBSAM/utredn/gellerstam .pdf, Rev. 2002

Harnesk, Jakob: Biblioteksservice för distansstuderande: en förstudie. Distansutbildnings-myndigheten, Rapport 2002:7

Hed, Helen: Bibliotekspedagogen - en ny profession? In: Gisselberg, Margareta: Distanslärare och distanslärande : en antologi. Distansutbildningsmyndigheten, Rapport 2002:6

HSV, Högskoleverket, http://www.hsv.se

Kommunaktuellt, 2000:40, S. 17

KULDA, http://www.kulda.se

Kühne, Brigitte: Biblioteket - skolans hjärna? Skolbiblioteket som resurs i det undersökande arbetssättet på grundskolan. Lund, 1993

Kühne, Brigitte: DERAL - Distance Education in Rural Areas via Libraries. Final Report - Deliverable D9.18. EC, Luxembourg, 2001

Lundborg, Bitte: Böckerna trängs undan av datorer, in: Kommunaktuellt, 2000:40, S. 16-17

Net University (Swedish: Nätuniversitetet), http://www.netuniversity.se

NITUS, http://www.nitus.org

Statens kulturråd, http://www.kur.se

Öppna högskolan. Prop. 2001/02:15. Stockholm, 2002

Die zitierten Internetadressen wurden zuletzt am 12.12.2004 aufgerufen. 